\title{
Is lipoprotein(a) a risk factor for ischemic stroke and venous thromboembolism?
}

\author{
Alexander Heinrich Nave $\mathrm{N}^{1,2,3,4}$ (D) Arnold von Eckardstein ${ }^{5}$
}

Published online: 5 March 2019

(c) The Author(s) 2019

\begin{abstract}
The structural similarity with plasminogen as well as thrombogenic and atherogenic in vitro functions raise the question if lipoprotein(a) (Lp(a)) is a risk factor for venous thromboembolism (VTE) and ischemic stroke. Numerous case-control and prospective studies using different cut-off values to define high Lp(a) generated conflicting evidence for both VTE and ischemic stroke. Several meta-analyses demonstrated independent associations of elevated Lp(a) with a history of VTE or ischemic stroke. However, the evidence of prospective studies for associations of Lp(a) with incident stroke or recurrent VTE remains inconclusive. For ischemic stroke, data suggest that $\mathrm{Lp}(\mathrm{a})$ increases the risk of large-artery atherosclerosis stroke, but not cardioembolic or lacunar stroke. Lp(a) may increase the risk of VTE in the presence of additional thrombophilic risk factors. Larger cohort studies are needed to elaborate the importance of higher Lp(a) cut-offs and interactions with other risk factors and subgroups of stroke or VTE. The value of $\operatorname{Lp}(a)$ to estimate residual vascular risk after the first thromboembolic event remains to be adequately explored.
\end{abstract}

Keywords Lipoprotein a $\cdot$ Atherosclerosis $\cdot$ Low-density lipoproteins $\cdot$ Ischemic stroke $\cdot$ Venous thrombosis

Lipoprotein(a) $[\mathrm{Lp}(\mathrm{a})]$ resembles to low-density lipoprotein (LDL) by the presence of one molecule apolipoprotein $B$ (apoB) and its relatively high content of cholesteryl esters in the core. These features lend several in vitro atherogenic properties to $\mathrm{Lp}(\mathrm{a})$ which are also exerted by LDL, for example proteoglycan binding and induction of foam cell formation [1]. Lp(a) differs from LDL by the presence of an additional apolipoprotein, termed apolipoprotein(a) (apo(a)), which is covalently bound to apoB by one disulfide bond. Apo(a) is homologous to plasminogen by

This article is part of the special issue "Lp(a) - Update 2018"

Alexander Heinrich Nave

alexander.nave@charite.de

Arnold von Eckardstein

arnold.voneckardstein@usz.ch

$1 \quad$ Klinik und Hochschulambulanz für

Neurologie, Charité-Universitätsmedizin Berlin,

Charitéplatz 1, 10117 Berlin, Germany

2 Center for Stroke Research Berlin (CSB),

Charité-Universitätsmedizin Berlin, Berlin, Germany

3 Berlin Institute of Health (BIH), Berlin, Germany

4 German Center for Cardiovascular Research (DZHK), partner site Berlin, Berlin, Germany

5 Institute of Clinical Chemistry, University and University Hospital of Zurich, Raemistraße 100, 8091 Zurich, Switzerland the presence of kringle IV and kringle $\mathrm{V}$ domains as well as a protease domain, which however is catalytically inactive. A variable number of repeat polymorphism of the kringle IV domain encodes for 40 apo(a) isoforms which differ by size and whose number is inversely correlated with Lp(a) plasma concentrations [2]. The similarity to plasminogen as well as the presence of oxidized phospholipids have been made responsible for the thrombogenic properties of $\mathrm{Lp}(\mathrm{a})$ which include the inhibition of fibrinolysis, the induction of plasminogen activator inhibitor type 1 (PAI-1) expression in endothelial cells, as well as the increasing of activity of tissue factor pathway inhibitor and platelet responsiveness [3].

These properties have led to the hypothesis that elevated plasma levels of $\mathrm{Lp}(\mathrm{a})$ increase the risk of ischemic stroke and venous thromboembolism (VTE).

\section{Stroke}

Stroke is an acute and potentially life-threatening disease caused by the loss of cerebral blood supply leading to focal cerebral ischemia and ultimately to cell death of the central nervous system [4]. Worldwide, stroke is considered the second most common cause of death and one of the leading causes of disability in adult life [5]. Opposed to hemorrhagic stroke (i.e. intracerebral hemorrhage), more than $80 \%$ of all stroke cases are ischemic stroke events and 
are caused by occlusion of brain-supplying arteries. Despite current secondary prevention strategies, the risk of recurrent stroke events remains high within the first years after stroke [6]. The heterogeneous etiology of ischemic stroke leads to the distinction of subgroups of ischemic stroke [7]. According to the most commonly used Trial of ORG 10172 in Acute Stroke Treatment (TOAST) classification [7], ischemic stroke can be caused by large-artery atherosclerosis, cardioembolic events, small artery occlusion (i.e. lacunar stroke), other causes (e.g. arterial dissection), or unknown (cryptogenic) or competing causes. Of note, in populationbased studies large-artery atherosclerosis represent $<20 \%$ of all stroke events, whereas cryptogenic strokes represent $30-40 \%$ [8]. High blood pressure, presence of atrial fibrillation, and dyslipidemia (among others) are established risk factors for ischemic stroke; however, the causal role of $\mathrm{Lp}(\mathrm{a})$ in ischemic stroke is controversial.

\section{Ischemic stroke and lipoprotein(a)}

A significant association between high Lp(a) levels and increased risk of ischemic stroke was expected for a long time and first clinical reports date back to the mid-1980s [9, 10]. However, a nested case-control study using prospectively collected plasma samples from the Physicians Health Study published by Ridker and colleagues in 1995 found no association between $\mathrm{Lp}(\mathrm{a})$ and the risk of future stroke among healthy, middle-aged men [11]. Despite the negative finding of this study, a great number of case-control and several prospective studies examining different cohorts have reported varying results since $[12,13]$.

So far, three meta-analyses [14-16] summarized the existing evidence regarding $\mathrm{Lp}(\mathrm{a})$ and stroke from both case-control and prospective studies. All three meta-analyses demonstrated a significant and independent association of elevated $\mathrm{Lp}(\mathrm{a})$ with increased risk of ischemic stroke. The largest meta-analysis performed by the Emerging Risk Factor Collaboration [15] analyzed individual patient data from 13 prospective studies comprising 1684 first-ever ischemic stroke patients. After adjustment for confounding variables, an increase per 3.5-fold (1 standard deviation) higher usual $\mathrm{Lp}$ (a) level resulted in an adjusted risk ratio of 1.11 (95\% confidence interval [95\% CI] 1.02 to 1.20) indicating $\mathrm{Lp}(\mathrm{a})$ to be a modest, continuous, and independent risk factor for ischemic stroke. No analyses were performed on specific ischemic stroke subgroups. The most recent meta-analysis assessed generic data of both prospective and observational studies and aimed to explore subgroup-specific risk differences [16]. The metaanalysis of nine prospective studies demonstrated an adjusted relative risk (RR) of 1.29 (95\% CI 1.06 to 1.58 ) for ischemic stroke when comparing high vs. low Lp(a) levels.
The analyses revealed a strong heterogeneity between the studies. In fact, studies that chose categorical cut-off values to define elevated Lp(a) levels (comparing very high vs. very low $\mathrm{Lp}(\mathrm{a})$ levels) reported greater relative risks for ischemic stroke, compared to studies analyzing continuous relationships (e.g. change in risk per 1 standard deviation increase in $\mathrm{Lp}$ (a) level). Furthermore, subgroup-specific analyses revealed that greater relative risks in studies investigating younger individuals. In line with this observation are previously published studies assessing the risk of high $\mathrm{Lp}(\mathrm{a})$ in pediatric stroke that report much higher risk ratios [17]. It may be argued that this fact is due to the low absolute risk that is present in younger stroke patients overall. The performed meta-regression in the latest meta-analyses attributed some of the observed heterogeneity among case-control studies to subtypes of ischemic stroke. Studies that investigated patients with large artery atherosclerosis or cryptogenic stroke demonstrated greater odds ratios than studies involving stroke patients of other cause. Thus, it seems to be important to investigate the role of $\mathrm{Lp}(\mathrm{a})$ in specific subgroups of ischemic stroke.

\section{Ischemic stroke subgroups}

Many previously published studies investigating the risk of stroke of patients with elevated levels of $\mathrm{Lp}(\mathrm{a})$ fail to report the underlying cause of the stroke event. As stroke etiologies vary substantially between cohorts, this fact may explain some of the inconsistent results reported in the literature. For example, the prospective Atherosclerosis Risk in Communities (ARIC) study examined 14,221 individuals free of cardiovascular disease and investigated the risk of ischemic stroke with high levels of $\mathrm{Lp}(\mathrm{a})$ defined as the 80th percentile of the cohort [18]. After an average follow-up of 13.4 years 531 ischemic stroke events had occurred. Comparing different stroke subtypes, high Lp(a) increased the adjusted risk for non-lacunar stroke (RR 1.42, $95 \%$ CI 1.10 to 1.84 ), but not for lacunar stroke (RR 1.16, $95 \%$ CI 0.75 to 1.80 ) or cardio-embolic stroke (RR 1.07, $95 \%$ CI 0.65 to 1.74 ). Similarly, a more recent analysis of the ARIC study found that $\mathrm{Lp}(\mathrm{a})$ levels $>50 \mathrm{mg} / \mathrm{dl}$ increase the relative risk of stroke by $42 \%$ among individuals without atrial fibrillation, but not among patients with present atrial fibrillation reinforcing a pathological role of $\mathrm{Lp}(\mathrm{a})$ primarily in atherothrombotic (atherosclerotic) stroke [19]. This assumption is supported by a previous Korean study where higher $\mathrm{Lp}$ (a) levels were found in patients with largeartery atherosclerotic stroke compared to patients with other stroke subtypes [20]. The authors also demonstrated a significant correlation of $\mathrm{Lp}$ (a) levels with the degree of intracranial and extracranial carotid stenosis. Further evidence is provided by a previous genetic study that investigated 
the association of ischemic stroke risk with two variants (rs10455872 and rs3798220) of the apolipoprotein (a) gene (LPA). Among 9396 stroke patients that were analyzed, the odds ratio per variant allele was 1.27 (95\% CI 1.11 to 1.46$)$ for large-artery atherosclerotic stroke, 1.03 (95\% CI 0.88 to 1.22) for cardioembolic stroke, and 1.06 (95\% CI 0.90 to 1.24) for lacunar stroke.

\section{Risk of recurrent ischemic events}

So far, only few studies have investigated the residual risk of first-ever ischemic stroke patients with high levels of Lp(a). One study comprising 250 first-ever, acute stroke patients found an increased risk for the composite endpoint of recurrent stroke, transient ischemic attack, myocardial infarction, revascularization procedures, or cardiovascular death after one year with high $\mathrm{Lp}(\mathrm{a})$ defined at a cut-off level of $30 \mathrm{mg} / \mathrm{dl}$ (adjusted odds ratio [OR] 2.60, 95\% CI 1.19 to 5.47) [21]. Interestingly, most of the recurrent vascular events occurred during the first months after the index stroke. Using the same cut-off level of $30 \mathrm{mg} / \mathrm{dl}$, a recent Chinese study demonstrated an increased risk of recurrent ischemic stroke events at follow-up three months after the index stroke (adjusted OR 2.58, 95\% CI 1.11 to 6.01) [22]. Future studies with larger cohorts are needed to investigate, if high Lp(a) levels increase the risk of recurrent vascular events, especially within the first months after the index event.

\section{Venous thromboembolism in adults}

The majority of studies which investigated the association of Lp(a) with VTE were cross-sectional rather than prospective. The most recent meta-analysis analyzed the data of ten studies encompassing 13,541 subjects of whom 5660 had a history of deep vein thrombosis and/or pulmonary embolism [23]. As the cut-off defining elevated Lp(a), the authors used the upper limit of the manufacturer's product reference range (usually $30 \mathrm{mg} / \mathrm{dL}$ ) in nine studies and the 75 th percentile of $\mathrm{Lp}$ (a) value in the control group in one study. With these definitions, elevated Lp(a) was associated with the presence of VTE at an odds ratio of $1.56(95 \% \mathrm{CI}$ 1.36 to 1.79 ). Much stronger risk associations were found in patients who have a very high risk of VTE, for example patients immobilized due to paraplegia. In a case-control study of 279 Chinese patients with spinal cord injury of whom 55 had a VTE, an Lp(a) level $>30 \mathrm{mg} / \mathrm{dL}$ was associated with a more than 10-fold increased risk of VTE (multivariate OR $10.35,95 \%$ CI 2.37 to 45.15 ) [24].

In contrast to case-control studies, the data of prospective studies are more controversial. Among 8960 partici- pants of the Copenhagen City Heart Study, of whom 735 experienced a VTE event during 15 to 18 years of followup, Kamstrup et al. did not find any significant association of Lp(a) levels with the incidence of VTE. Adjusted hazard ratios for second (median, IQR: 17, 12-27 mg/dl) and third tertiles (median, IQR: 59, 40-94 mg/dl) vs. first tertile of Lp(a) (median, IQR: 3,1-5 mg/dl) were 1.1 (95\% CI $0.8-1.4)$ and 0.8 (95\% CI 0.6-1.1), respectively [25]. Likewise, in a 12-center study of 510 patients with first unprovoked VTE treated for 5-7 months with anticoagulants and followed up for $16.9 \pm 11.2$ months, Rodgers et al. did not find any significant association of $\mathrm{Lp}$ (a) levels $>300 \mathrm{mg} / \mathrm{L}$ with risk of recurrent VTE events (relative risk 1.4, 95\% CI 0.7-2.6) [26]. Conversely, in a study of 467 patients with first VTE followed up for one year, Marcucci et al. found a 5-fold increased risk of recurrent VTE for $\mathrm{Lp}(\mathrm{a})$ $>30 \mathrm{mg} / \mathrm{dL}$ (OR 5.1, 95\% CI 3.1-8.4) which was similar to that for hyperhomocysteinemia and even higher than that for factor V Leiden or the factor II 20210GA polymorphism [27].

Mendelian randomization studies also made controversial findings on the association of LPA polymorphisms with risk of VTE. Kamstrup and colleagues [25] excluded any contribution of the kringle IV repeat polymorphism to VTE in the Copenhagen City Heart Study ( $N=9190,443$ events) and the Copenhagen General Population study $(N=28,538$; 926 with history of VTE). Of note, in the same study, the authors found genetically causal associations of Lp(a) levels and kringle IV repeats with coronary, carotid and femoral atherosclerosis as well as of factor V Leiden with VTE [25]. A more recent but smaller study of 516 patients with a history of VTE and 1117 controls found significant inverse and dose-dependent associations of kringle IV repeat numbers with venous thrombosis [28].

\section{Venous thromboembolism in children and adolescents}

With an annual incidence of less than 1 in 100,000, VTE is a rare condition in childhood and adolescence. Nevertheless, several studies have investigated the associations of $\mathrm{Lp}(\mathrm{a})$ and other thrombophilic risk factors with the presence and recurrent incidence of VTE in this young population.

A meta-analysis of eight studies encompassing 589 patients with VTE and 1441 controls, Lp(a) levels $>300 \mathrm{mg} / \mathrm{L}$ was associated with a 4.5 -fold higher risk of first VTE $(95 \%$ CI 3.19 to 6.35) [29]. One of the studies included also investigated the association of the kringle IV repeat polymorphism with VTE. In 186 patients with VTE and 186 matched controls the highest quartile of $\mathrm{Lp}(\mathrm{a})$ levels and the lowest quartile of kringle IV repeats were associated with similar risks of VTE (7.68 vs. 8.14) [30]. Also the 
history of cerebral venous thrombosis in childhood was associated with elevated Lp(a) significantly (multivariate OR 4.1; 95\% CI 1.9-8.6) and even more strongly than with factor V Leiden or the 20210GA polymorphism [31].

A meta-analysis of six studies with 135 recurrent VTEs among 1155 children and adolescents followed up for a median time of 48 months (minimum, 12 months; maximum, 96 months) after the first VTE did not find any significant association of high $\mathrm{Lp}$ (a) levels with the risk of recurrent VTE events (OR 0.84; 95\% CI 0.5 to 1.4). However, in this meta-analysis neither factor $\mathrm{V}$ Leiden nor the prothrombin polymorphism were significantly associated with the risk of recurrent VTE. Only the combination of at least two thrombophilic risk factors including elevated $\mathrm{Lp}(\mathrm{a})$ evolved as a prognostic risk factor for recurrent VTE (OR 4.91; $95 \%$ CI 3.12 to 7.74) [29]. One of the studies followed up 301 young patients with start of anticoagulation within 6 months after first VTE and assessed 54 recurrent VTEs within 6 months to 15 years of follow-up [32]: Elevated $\mathrm{Lp}$ (a) per se did not significantly increase the risk of recurrent VTE (OR,: 1.35; 95\% CI,: 0.85-2.13). However, the combination of $\mathrm{Lp}$ (a) $>300 \mathrm{mg} / \mathrm{L}$ with at least one other thrombophilic risk factor (factor $\mathrm{V}$ Leiden, prothrombin 20210GA, MTHFR 677TT, protein C deficiency, protein S deficiency, or antithrombin deficiency) increased the risk of VTE by factor 2.36 (95\% CI 1.51-3.68) [32]. The strongest risk factor was the combination of elevated $\mathrm{Lp}(\mathrm{a})$ with factor V Leiden which increased the risk of recurrent VTE by factor 6.2 [32].

\section{Conclusions}

Lp(a) has been shown to be an independent and modest risk factor for ischemic stroke in recent meta-analyses of prospective studies. Overall, the relative risk is increased by approximately $30 \%$; however, the reported differences vary according to the examined population including stroke subtype and the applied statistical methodology. Data suggest that $\mathrm{Lp}(\mathrm{a})$ is primarily increasing the risk of large-artery atherosclerosis stroke. Nevertheless, controversial literature and limited therapeutic options detain current guidelines to recommend the routine measurement of $\mathrm{Lp}(\mathrm{a})$ after stroke [33].

The role of $\mathrm{Lp}(\mathrm{a})$ as a thrombophilic risk factor remains even more equivocal: the meta-analyses of cross-sectional studies revealed significant associations of $\mathrm{Lp}(\mathrm{a})>30 \mathrm{mg} / \mathrm{dl}$ with a history of VTE. However, data of genetic studies on the associations of kringle IV repeats with VTE as well as of prospective studies on associations of high Lp(a) levels with recurrent VTE are controversial. Possibly, elevated $\mathrm{Lp}$ (a) becomes a relevant thrombophilic risk factor if it coincides with other hereditary thrombophilic risk factors and/or thrombophilic basal conditions (e.g. long-term immobilization).

Larger cohort studies are necessary to test whether higher cut-off values than conventional $30 \mathrm{mg} / \mathrm{dl}$ or $50 \mathrm{mg} / \mathrm{dl}$, interactions with other risk factors, or etiological disease differentiation are needed to firmly establish or rule-out any role of $\mathrm{Lp}(\mathrm{a})$ as a risk factor of ischemic stroke or VTE.

Conflict of interest A.H. Nave and A. von Eckardstein declare that they have no competing interests.

Open Access This article is distributed under the terms of the Creative Commons Attribution 4.0 International License (http:// creativecommons.org/licenses/by/4.0/), which permits unrestricted use, distribution, and reproduction in any medium, provided you give appropriate credit to the original author(s) and the source, provide a link to the Creative Commons license, and indicate if changes were made.

\section{References}

1. Gencer B, Kronenberg F, Stroes ES, Mach F (2017) Lipoprotein(a): the revenant. Eur Heart J 38:1553-1560. https://doi.org/10.1093/ eurheartj/ehx033

2. Schmidt K, Noureen A, Kronenberg F, Utermann G (2016) Structure, function, and genetics of lipoprotein (a). J Lipid Res 57:1339-1359. https://doi.org/10.1194/jlr.R067314

3. Boffa MB, Koschinsky ML (2016) Lipoprotein (a): truly a direct prothrombotic factor in cardiovascular disease? J Lipid Res 57:745-757. https://doi.org/10.1194/jlr.R060582

4. Sacco RL, Kasner SE, Broderick JP et al (2013) An updated definition of stroke for the 21 st century: a statement for healthcare professionals from the American heart association/American stroke association. Stroke 44:2064-2089. https://doi.org/10.1161/ STR.0b013e318296aeca

5. Feigin VL, Norrving B, Mensah GA (2017) Global burden of stroke. Circ Res 120:439-448. https://doi.org/10.1161/CIRCRE SAHA.116.308413

6. Amarenco P, Lavallée PC, Monteiro Tavares L et al (2018) Fiveyear risk of stroke after TIA or minor Ischemic stroke. N Engl J Med 378:2182-2190. https://doi.org/10.1056/NEJMoa1802712

7. Adams HP, Bendixen BH, Jaap Kappelle L et al (1993) Classification of subtype of acute Ischemic stroke definitions for use in a multicenter clinical trial. Stroke 24:35-41

8. Li L, Yiin GS, Geraghty OC et al (2015) Incidence, outcome, risk factors, and long-term prognosis of cryptogenic transient ischaemic attack and ischaemic stroke: A population-based study. Lancet Neurol 14:903-913. https://doi.org/10.1016/S1474-4422(15)00132-5

9. Költringer P, Jürgens G (1985) A dominant role of lipoprotein(a) in the investigation and evaluation of parameters indicating the development of cervical atherosclerosis. Atherosclerosis 58:187-198. https://doi.org/10.1016/0021-9150(85)90065-6

10. Zenker G, Költringer P, Boné G et al (1986) Lipoprotein(a) as a strong indicator for cerebrovascular disease. Stroke 17:942-945. https://doi.org/10.1161/01.STR.17.5.942

11. Ridker PM, Stampfer MJ, Hennekens CH (1995) Plasma concentration of lipoprotein(a) and the risk of future stroke. JAMA 273:1269-1273

12. Danik SJ, Rifai N, Buring JE, Ridker PM (2006) Lipoprotein(a), measured with an assay independent of apolipoprotein(a) isoform size, and risk of future cardiovascular events among initially healthy women. JAMA 296:1363-1370. https://doi.org/10.1001/jama.296. 11.1363 
13. Gurdasani D, Sjouke B, Tsimikas S et al (2012) Lipoprotein(a) and risk of coronary, cerebrovascular, and peripheral artery disease: the EPIC-Norfolk prospective population study. Arterioscler Thromb Vasc Biol 32:3058-3065. https://doi.org/10.1161/ATVBAHA.112. 255521

14. Smolders B, Lemmens R, Thijs V (2007) Lipoprotein (a) and stroke: a meta-analysis of observational studies. Stroke 38: 1959-1966. https://doi.org/10.1161/STROKEAHA.106.480657

15. Erqou S, Kaptoge S, Perry P et al (2009) Lipoprotein(a) concentration and the risk of coronary heart disease, stroke, and nonvascular mortality. JAMA 302:412-423

16. Nave AHAH, Lange KSKS, Leonards COCO et al (2015) Lipoprotein (a) as a risk factor for ischemic stroke: a meta-analysis. Atherosclerosis 242:496-503. https://doi.org/10.1016/j.atheroscle rosis.2015.08.021

17. Sträter R, Becker S, von Eckardstein A et al (2002) Prospective assessment of risk factors for recurrent stroke during childhood-a 5-year follow-up study. Lancet 360:1540-1545. https://doi.org/10. 1016/S0140-6736(02)11520-0

18. Ohira T, Shahar E, Chambless LE et al (2006) Risk factors for ischemic stroke subtypes: the atherosclerosis risk in communities study. Stroke 37:2493-2498. https://doi.org/10.1161/01.STR. 0000239694.19359.88

19. Aronis KN, Di Zhao, Hoogeveen RC et al (2017) Associations of Lipoprotein(a) levels with incident atrial fibrillation and Ischemic Stroke: the ARIC (Atherosclerosis Risk in Communities) study. J Am Heart Assoc 6:1-11. https://doi.org/10.1161/JAHA. 117.007372

20. Kim BS, Jung HS, Bang OY et al (2010) Elevated serum lipoprotein(a) as a potential predictor for combined intracranial and extracranial artery stenosis in patients with ischemic stroke. Atherosclerosis 212:682-688. https://doi.org/10.1016/j.atheroscle rosis.2010.07.007

21. Lange KS, Nave AH, Liman TG et al (2017) Lipoprotein(a) levels and recurrent vascular events after first Ischemic stroke. Stroke 48:36-42. https://doi.org/10.1161/STROKEAHA.116.014436

22. Hong X-W, Wu D-M, Lu J et al (2018) Lipoprotein (a) as a predictor of early stroke recurrence in acute Ischemic stroke. Mol Neurobiol 55:718-726. https://doi.org/10.1007/s12035-016-0346-9

23. Dentali F, Gessi V, Marcucci R et al (2017) Lipoprotein(a) as a risk factor for venous thromboembolism: a systematic review and meta- analysis of the literature. Semin Thromb Hemost 43:614-620. https://doi.org/10.1055/s-0036-1598002

24. Wang C-W, Su L-L, Tao S-B et al (2016) An increased serum level of lipoprotein(a) is a predictor for deep vein thrombosis in patients with spinal cord injuries. World Neurosurg 87:607-612. https://doi. org/10.1016/j.wneu.2015.10.059

25. Kamstrup PR, Tybjærg-Hansen A, Nordestgaard BG (2012) Genetic evidence that lipoprotein(a) associates with atherosclerotic stenosis rather than venous thrombosis. Arterioscler Thromb Vasc Biol 32:1732-1741. https://doi.org/10.1161/ATVBAHA.112. 248765

26. Rodger MA, Le Gal G, Carrier M et al (2010) Serum lipoprotein (a) levels in patients with first unprovoked venous thromboembolism is not associated with subsequent risk of recurrent VTE. Thromb Res 126:222-226. https://doi.org/10.1016/j.thromres.2010.06.007

27. Marcucci R, Liotta AA, Cellai AP et al (2003) Increased plasma levels of lipoprotein(a) and the risk of idiopathic and recurrent venous thromboembolism. Am J Med 115:601-605

28. Sticchi E, Magi A, Kamstrup PR et al (2016) Apolipoprotein(a) Kringle-IV type 2 copy number variation is associated with venous thromboembolism. PLoS ONE 11:e149427. https://doi.org/ 10.1371/journal.pone.0149427

29. Young G, Albisetti M, Bonduel M et al (2008) Impact of inherited thrombophilia on venous thromboembolism in children. Circulation 118:1373-1382. https://doi.org/10.1161/CIRCULATIONAHA. 108.789008

30. Nowak-Göttl U, Junker R, Hartmeier M et al (1999) Increased lipoprotein(a) is an important risk factor for venous thromboembolism in childhood. Circulation 100:743-748. https://doi.org/10. 1016/s0140-6736(05)80081-9

31. Heller C, Heinecke A, Junker R et al (2003) Cerebral venous thrombosis in children: A multifactorial origin. Circulation 108: 1362-1367. https://doi.org/10.1161/01.CIR.0000087598.05977.45

32. Nowak-Göttl U, Junker R, Kreuz W et al (2001) Risk of recurrent venous thrombosis in children with combined prothrombotic risk factors. Blood 97:858-862. https://doi.org/10.1182/blood.V97. 4.858

33. Yaghi S, Elkind MSV (2015) Lipids and Cerebrovascular Disease. Stroke 46:3322-3328. https://doi.org/10.1161/STROKEAHA.115. 011164 